\title{
Numerical Modeling of Nitrogen Absorption during Gas Tungsten Arc Welding
}

\author{
by Kazuki SUGIURA**, Shinji KODAMA***, Yoshihiro TSUJIMURA****, \\ Anthony Bruce MURPHY***** and Manabu TANAKA****
}

\begin{abstract}
It is problem that nitrogen absorption in gas tungsten arc (GTA) weld metal. Recently, various arc welding simulation techniques have been developed. However, there are few models dealing with the absorption and a mixture of a shielding gas, an atmosphere and a metal vapor.. In this study, intruding behaviors of the atmospheric gas $\left(\mathrm{N}_{2}\right)$ into the molten poor and nitrogen transportation phenomenon in the molten poor during GTA welding was investigated using a unified numerical model. This model includes the tungsten cathode, arc plasma and base metal. We take atmosphere $\left(\mathrm{N}_{2}\right)$, shielding gas from the gas nozzle and metal vapor from the molten poor into consideration. As a result, we show nitrogen concentration distribution and nitrogen transportation in the molten poor. Additionally, we clarify that these behaviors are affected by the arc plasma characteristics.
\end{abstract}

Key Words: $\quad$ Numerical Model, Gas Tungsten Arc Welding, Nitrogen, Absorption

\section{Introduction}

Nitrogen absorption in weld metal causes deterioration of welding quality, such as blowhole generation or toughness degradation. According to previous studies, it is known that weld metals may generate blowholes when the nitrogen concentration exceed around 200ppm, and the toughness begins to degrade sharply as it surpasses around $100 \mathrm{ppm}^{1)}$.Meanwhile, various arc welding simulation techniques have been developed. However, in most of the conventional arc welding models, the atmosphere is treated the same as the shielding gas, and thus there are few models in which the mixture of atmospheric nitrogen is taken into consideration. Furthermore, the model which calculates the nitrogen absorption in the molten pool hardly exists. In this paper, the numerical-analysis model of GTA welding was developed for analysing the mixing behaviour of the atmospheric nitrogen in the shielding gas and dissolving phenomenon of the atomic nitrogen into molten pool. By using this model, the nitrogen transportation in the molten pool was calculated.

\section{The numerical-analysis model of GTA welding}

The atmospheric nitrogen is mixed in the shielding gas under the influence of the arc jet flow, and the nitrogen is

\footnotetext{
${ }^{*}$ Received: 2012.11 .29

${ }^{* *}$ Student Member, Graduate School of Engineering, Osaka University

${ }^{* * *}$ Member, NIPPON STEEL \& SUMITOMO METAL CORPORATION

${ }^{* * * *}$ Member, Joining and Welding Research Institute, Osaka university

Member, CSRIO Materials Science and Engineering
}

disassociated in the plasma region of high temperature. Furthermore, the disassociated nitrogen dissolves in the molten metal surface, and it spreads through the molten metal by the convection flow and diffusion of molten pool. In other to calculate this intricate phenomenon, the mixture phenomenon of nitrogen and shielding gas, the nitrogen dissolution behaviour on molten pool surface, and the nitrogen transfer in molten pool were calculated simultaneously.

The simulation domain is shown in Fig.1. The two-dimensional cylindrical coordination model, which consists of a tungsten cathode having a diameter of $3.2 \mathrm{~mm}$ with a tip angle of $60^{\circ}$, a pure iron anode having a diameter of $25 \mathrm{~mm}$ and a shielding nozzle with an inner diameter of $12 \mathrm{~mm}$, was adopted. Arc length is set as $5 \mathrm{~mm}$. In addition, the current were set at $150 \mathrm{~A}$. Moreover, nitrogen was postulated as the atmosphere

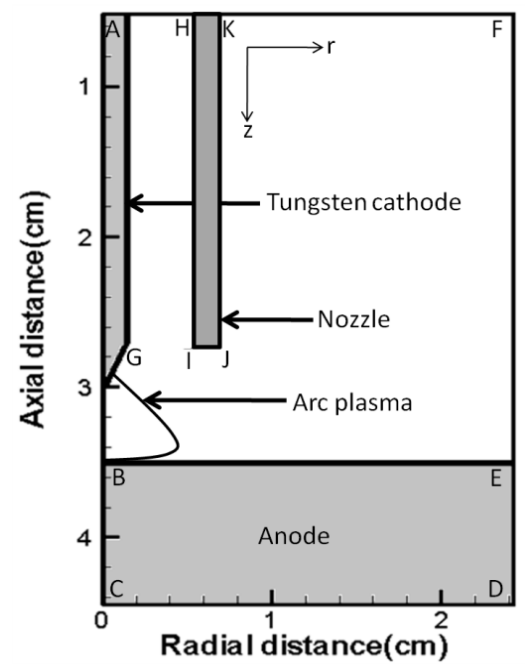

Fig. 1. Schematic illustration of simulation domain. 
In this model, mass conservation equation, momentum conservation equation, energy conservation equation and current conservation equation were used as the dominant equations. The mixture of the shielding gas (helium), the atmosphere (nitrogen) and the iron vapor from the molten pool was analyzed using such a conservation equation ${ }^{2}$.

$$
\frac{\partial}{\partial t}\left(\rho C_{1}\right)+\frac{1}{r} \frac{\partial}{\partial r}\left(r \rho_{r} G_{1}\right)+\frac{\partial}{\partial z}\left(\rho_{z} G_{1}\right)=\frac{1}{r} \frac{\partial}{\partial r}\left(r \rho D \frac{\partial C_{1}}{\partial r}\right)+\frac{\partial}{\partial z}\left(\rho D \frac{\partial C_{1}}{\partial z}\right)
$$

where, $C_{1}$ is the mass fraction of each elements, $v r$ and $v z$ are the respective gas flow velocity in the radial direction and the axial direction, and $\rho$ is density. Furthermore, $D$ is the two-dimensional diffusion coefficient which is expressed by the viscosity approximation equation

$$
D=\frac{2 \sqrt{2}\left(1 / M_{1}+1 / M_{2}\right)^{0.5}}{\left[\left(\rho_{1}^{2} / \beta_{1}^{2} \mu_{1}^{2} M_{1}\right)^{0.25}+\left(\rho_{2}^{2} / \beta_{2}^{2} \mu_{2}^{2} M_{2}\right)^{0.25}\right]^{2}}
$$

where M1 and M2 are the molecular weights of iron and the shielding gas respectively. $\rho 2, \rho 2, \eta 1$ and $\eta_{2}$ are the density and viscosity of iron and the shielding gas respectively. $\beta 1$ and $\beta 2$ are the dimensionless constants. $\beta 1=\beta 2=1.385$ is assumed based on the mean value of the experimental data ${ }^{3)}$.

The nitrogen concentration of the surface of a molten pool was defined by the following formula ${ }^{4)}$.

$$
[N]=P_{N} \exp \left(-\frac{\Delta G_{N_{(g)}}^{0}}{R T}\right)
$$

Where, $N$ is the nitrogen concentration, $P_{N}$ is the monatomic nitrogen partial pressure, $\Delta G_{N(g)}^{0}$ is the free energy relationship

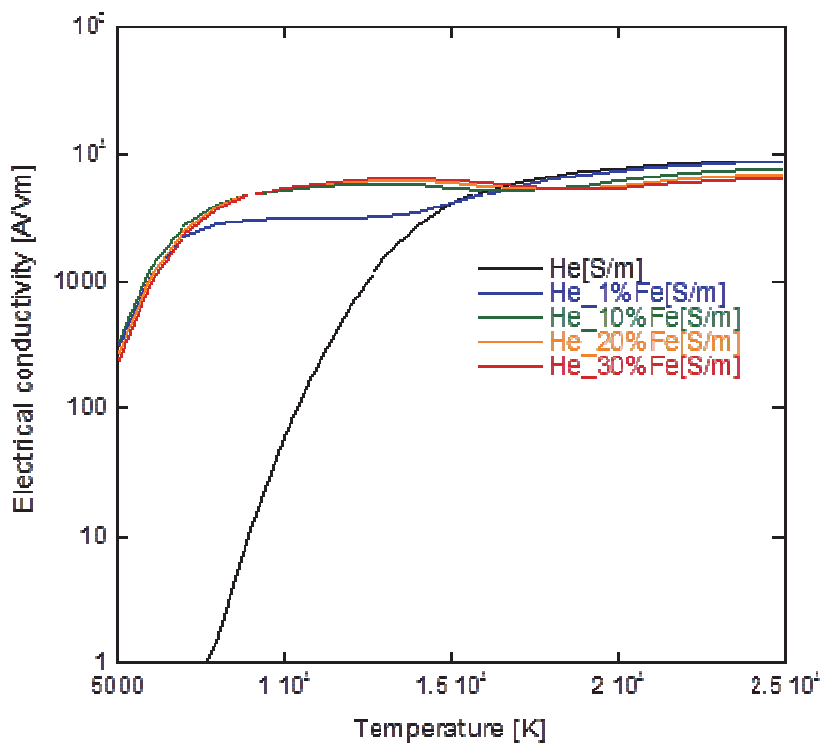

Fig. 2 Dependence of electrical conductivities of helium gas on temperature for each mixing ratio for the nitrogen absorption reactions. It turns out to be that the amount of absorption of nitrogen increases by the increase in $P_{N}$, and decrease of $T$ from this formula. The nitrogen concentration inside the molten pool was calculated by the convection flow and diffusion of nitrogen which defined by equation (1). The initial value of the nitrogen concentration of a base material was set at $30 \mathrm{ppm}$.

In the present model, plasma properties are dependent on not only the temperature but also the mole fraction of iron vapor and nitrogen. The plasma properties at the intermediate concentrations of iron vapor are calculated using a linear approximation based on the properties at $0,1,10,20$ and 30 mol.- $\%$ of iron vapor mixture $\operatorname{rate}^{5)}$. The properties were calculated using the Chapman-Enskog approximation ${ }^{6)}$ under the assumption that the arc plasma is in a local thermodynamic equilibrium (LTE) condition. All of electron temperature, ion temperature and heavy particle temperature are the same in the LTE condition. For example, the electrical conductivities and the radiative emission coefficients, which are significantly affected by the mixture of iron vapor, are shown in Figs. 2 and 3 respectively. As shown in Fig. 2 concerning the change in electrical conductivity of helium plasma, electrical conductivity greatly increases by the addition of iron vapor at the temperatures below $15000 \mathrm{~K}$ and the effect of the iron vapor addition does not differ between 1, 10, 20 and $30 \%$ of the mixing ratio. Figure 3 shows the change in the radiative emission coefficient. The radiative emission coefficient increases with increased mixing ratio of iron vapor while it remains in a very low level for pure helium plasma.

The governing and auxiliary equations were solved iteratively by the SIMPLEC numerical procedure.

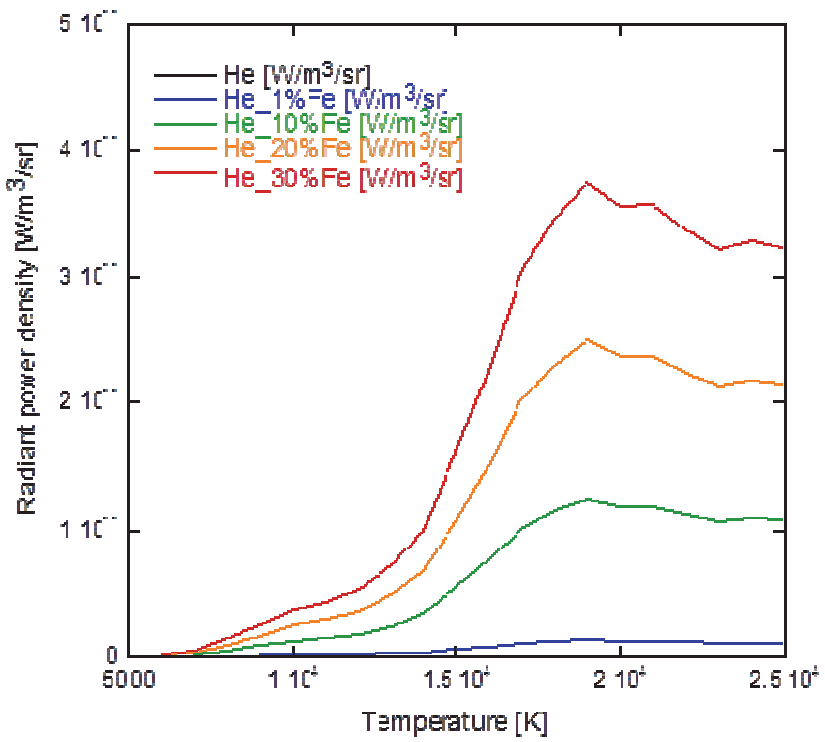

Fig. 3 Dependence of radiant power density of helium gas on temperature for each mixing ratio 


\section{Result and discussion}

Fig. 4 shows temperature distribution in the arc plasma and in the base metal. Fig.5 shows concentration distribution of helium in the arc plasma. Fig. 6 shows the mole fraction of iron vapor. The distribution of iron vapor is determined by the diffusion term and the convection term as described in equation (1). It is seen that distribution of iron vapor expands in the radial direction, concentrating just above the weld pool surface. The concentration of iron vapor mixed in arc plasma attains to $20 \mathrm{~mol} .-\%$.

Fig.7 shows the fluid flow path in the arc plasma and the molten pool. In an arc atmosphere where high speed plasma flow of $300 \mathrm{~m} / \mathrm{s}$ exists, the convection term dominates. In the molten pool, the nitrogen concentration in the molten pool is dominated

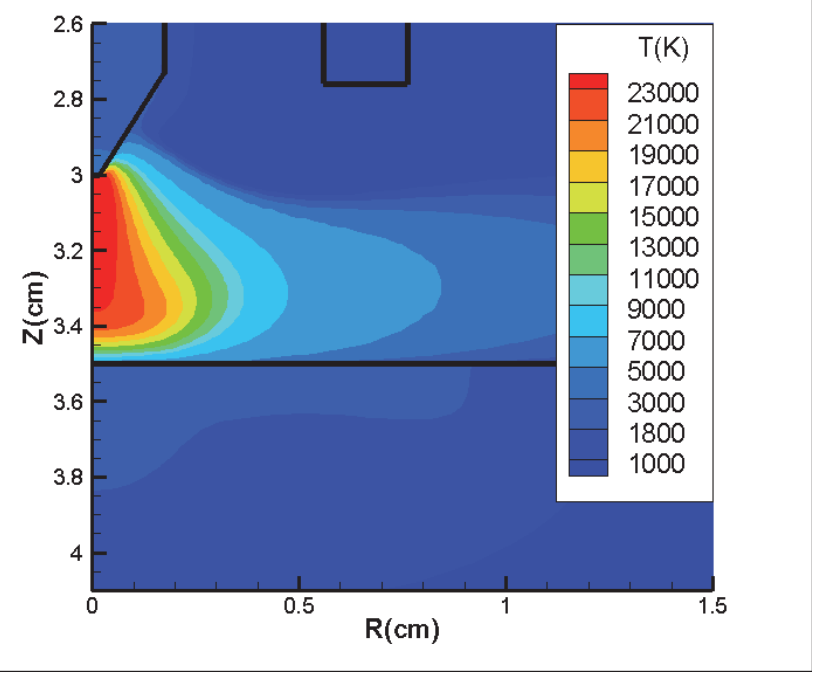

Fig.4 Temperature distribution in the arc plasma and in the base metal

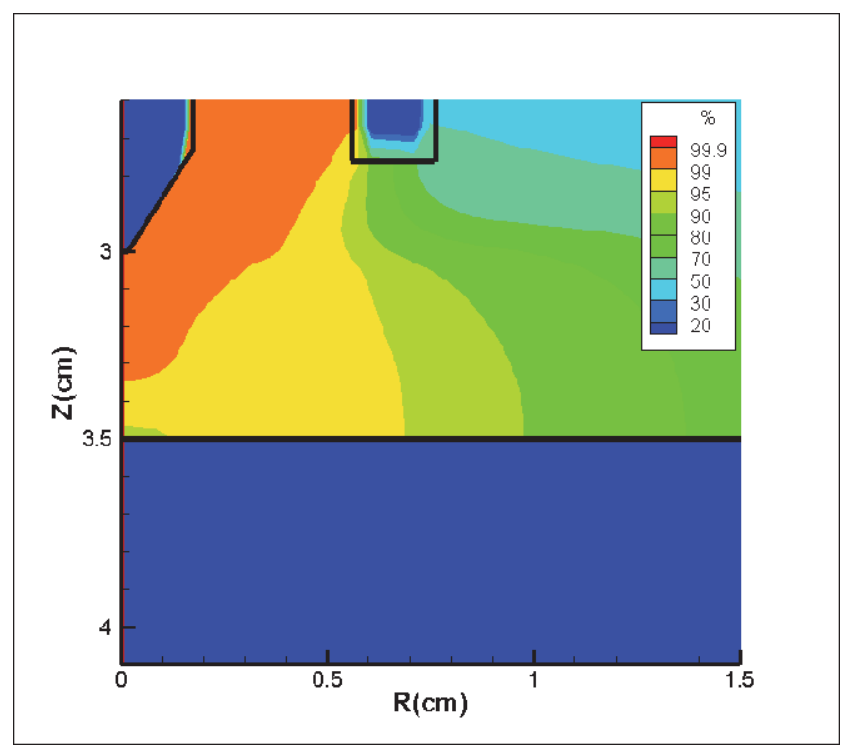

Fig. 5 Concentration distribution of helium in the arc plasma by two whirlpools. It is thought that drag and Marangoni force were mainly dominating the radial flow and electromagnetic force was mainly dominating the flow of the depth direction. The calculated maximum velocities for radial and vertical directions were $43 \mathrm{~cm} / \mathrm{sec}$ at the surface of the pool and $14.6 \mathrm{~cm} / \mathrm{sec}$ at the center of the pool.

Fig. 8 shows the concentration distribution of nitrogen in the arc plasma and in the base metal for each flow rate. The nitrogen concentration of the domain except a molten pool was $30 \mathrm{ppm}$. In the condition of arc ignition, the position of the maximum nitrogen concentration is located at the place away from the center of molten pool surface. In the central part of the molten pool surface, the nitrogen concentration decreases because the partial pressure of atomic nitrogen reduces caused by the metal

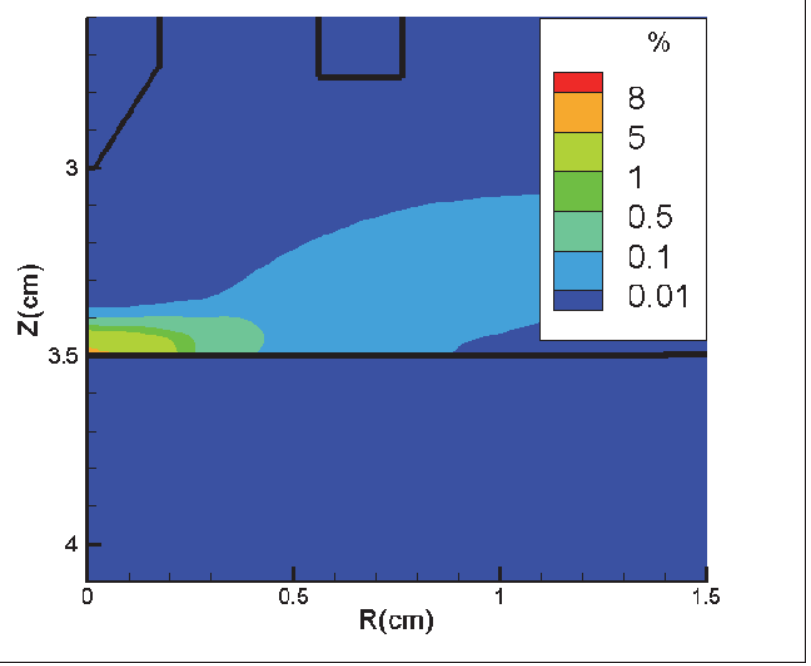

Fig. 6 Concentration distribution of Fe metal vapor in the arc plasma

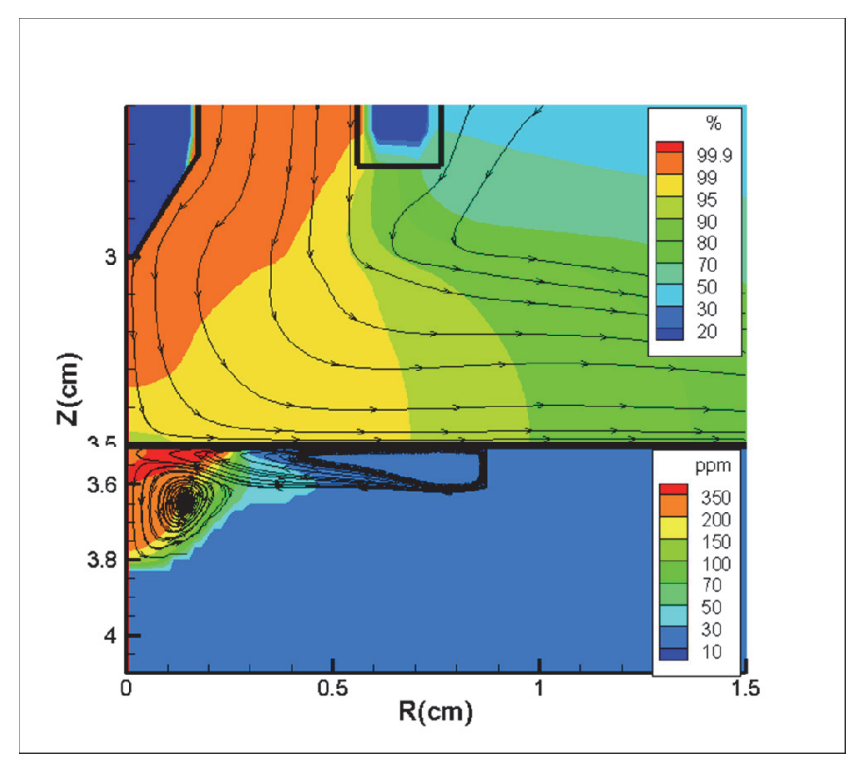

Fig. 7 Fluid flow path in the arc plasma and in the molten pool. 


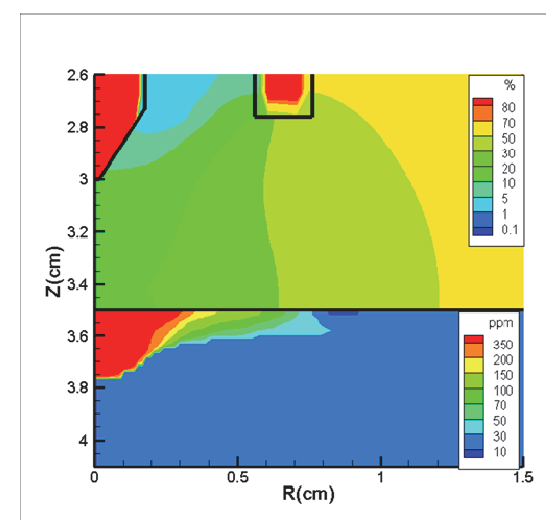

(a)

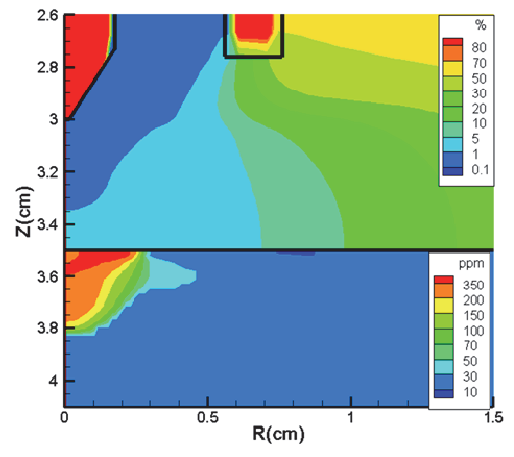

(b)

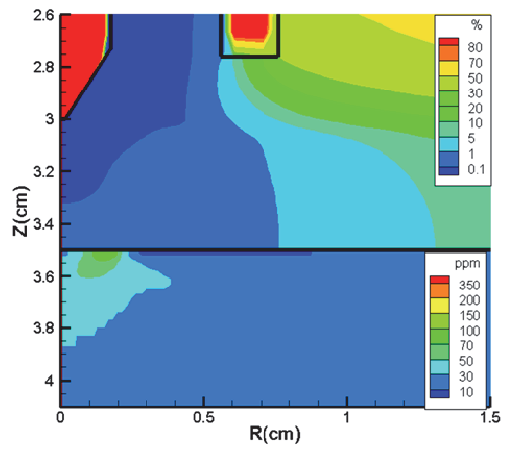

(c)

Fig. 8 Concentration distribution of nitrogen in the arc plasma and inside the molten pool

Shielding gas flow is (a) $5 \mathrm{~L} / \mathrm{min}$ (b) $10 \mathrm{~L} / \mathrm{min}$ (c) $20 \mathrm{~L} / \mathrm{min}$.

vapor generation, and the nitrogen absorption in molten pool is inhibited with the increase of the molten pool surface temperature. There were two whirlpools in the molten pool. The one is near the surface and another is near the center of the pool. Most nitrogen seemed to be transported to the depth direction by the whirlpool near the center. The other was seemed to be transported to the circumferential direction by the whirlpool near the surface, and emitted from a peripheral zone. The concentration of nitrogen in the arc plasma became larger when the shielding gas flow decreases. In the same way, the nitrogen concentration inside the molten pool increases dramatically with the decrease of shielding gas flow rate.

\section{Conclusions}

The numerical model of the GTA welding was developed, and the nitrogen concentration distribution and the transportation phenomenon in the molten pool were analyzed. The main conclusions are summarized as follows.

1) The position of the maximum nitrogen concentration existed in the place away from the molten pool surface center.

2) In the molten pool, the nitrogen concentration in the molten pool is dominated by two whirlpools. Most nitrogen seemed to be transported to the depth direction by the whirlpool near the center.
The other was seemed to be transported to the circumferential direction by the whirlpool near the surface, and emitted from a peripheral zone. The calculated maximum velocities for radial and vertical directions were $43 \mathrm{~cm} / \mathrm{sec}$ at the surface of the pool and $14.6 \mathrm{~cm} / \mathrm{sec}$ at the center of the pool.

3) The concentration of nitrogen inside the molten pool increased dramatically as a shielding gas flow rate decreases.

\section{Acknowledgements}

This work was supported by JSPS KAKENHI "Grant-in-Aid for Scientific Research (A)" Grant Number 22246095.

\section{Reference}

1) The report of research about the shielding performance of gas shielded arc welding. WELDING TECHNOLOGY. Volume 10, Number 57(2009)

2) J. Menart and L. Lin, Numerical Study of a Free-Burning Argon Arc with Copper Contamination from Anode, Plasma Chem Plasma Process. , 19-2 (1999), 153-170.

3) C.R. Wilke: A viscosity equation for gas mixtures, J. Chem. Phys., 18-4 (1950), 517-519.

4) T.A. PALMER and T. DEBROY, Numerical Modeling of Enhanced Nitrogen Dissolution during Gas Tungsten Arc Welding. METALLURGICAL AND MATERIALS TRANSACTIONS B. Volume 31, Number 6 (2000), 1371-1385

5) H. G. Fan, H. L. Tsai and S. J. Na: Int. J. Heat Mass Transfer, 2001, $44,417-428$

6) A. B.Murphy: Plasma Chem. Plasma Process., 1995, 15, (2), 279-307. 